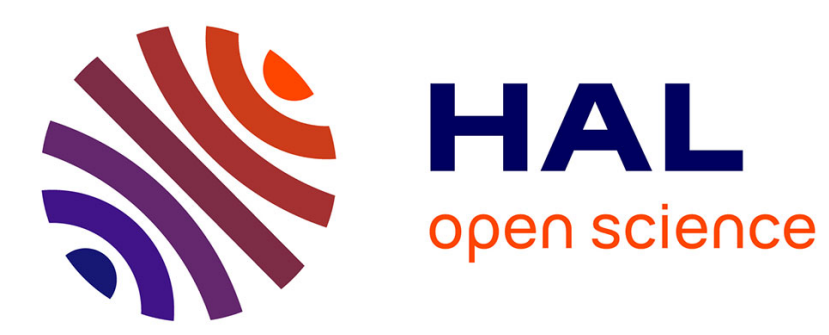

\title{
EXPERIMENTAL STUDIES OF ENERGY BAND STRUCTURE OF CHALCOPYRITE CRYSTALS
}

\author{
J. Shay
}

\section{To cite this version:}

J. Shay. EXPERIMENTAL STUDIES OF ENERGY BAND STRUCTURE OF CHALCOPYRITE CRYSTALS. Journal de Physique Colloques, 1975, 36 (C3), pp.C3-109-C3-113. 10.1051/jphyscol:1975321 . jpa-00216291

\section{HAL Id: jpa-00216291 https://hal.science/jpa-00216291}

Submitted on 1 Jan 1975

HAL is a multi-disciplinary open access archive for the deposit and dissemination of scientific research documents, whether they are published or not. The documents may come from teaching and research institutions in France or abroad, or from public or private research centers.
L'archive ouverte pluridisciplinaire HAL, est destinée au dépôt et à la diffusion de documents scientifiques de niveau recherche, publiés ou non, émanant des établissements d'enseignement et de recherche français ou étrangers, des laboratoires publics ou privés. 


\title{
EXPERIMENTAL STUDIES OF ENERGY BAND STRUCTURE OF CHALCOPYRITE CRYSTALS
}

\author{
J. L. SHAY
}

Bell Laboratories, Homldel, New Jersey 07733, USA

\begin{abstract}
Résumé. - Dans cet article, nous passons en revue les études expérimentales de la structure de bandes de cristaux des types II-IV-V ${ }_{2}$ et I-III-VI ${ }_{2}$ Les sujets présentant un intérêt pour des études ultérieures sont discutés.
\end{abstract}

Abstract. - In this paper we review experimental studies of the energy band structure of II-IV-V 2 and I-III-VI 2 crystals. Topics of interest for further study are discussed.

1. Introduction. - Ternary chalcopyrite II-IV-V and I-III-VI $\mathrm{V}_{2}$ semiconductors are the closest electronic and structural analogs of the zincblende III-V and IIVI semiconductors respectively. Experimental studies of the energy band structure of these ternary compounds prior to 1972 have been reviewed elsewhere [15]. In this work we review experimental studies since 1972 and discuss topics of interest for further study. In Section 2 a new interpretation of the electroreflectance spectrum of the pseudodirect crystal $\mathrm{ZnSiP}_{2}$ is presented, and experiments to substantiate the interpretation are proposed. In Section 3 we describe recent X-ray photoemission studies of the valence bands of I-III-VI ${ }_{2}$ compounds which have confirmed in detail the importance of noble-metal d-levels in the valence bands. The need for energy band calculations including these $\mathrm{d}$-levels is emphasized.

2. II-IV-V $\mathrm{V}_{2}$ compounds. - There are two qualitatively different types of energy band structures of II-IV $-V_{2}$ compounds. They correspond to ternary compounds analogous to direct-band-gap and indirectband-gap III-V compounds respectively. The band structure of $\mathrm{ZnGeAs}_{2}$, the analog of the direct-bandgap compound GaAs, is shown in figure 1. The band structure of $\mathrm{ZnGeP}_{2}$, the analog of the indirect-bandgap compound GaP, is shown in figure 2. The theoretical energy levels (indicated by circles in figures 1 and 2) were calculated by the pseudopotential method without spin-orbit coupling at the high symmetry points $\Gamma, \mathrm{T}$, $\mathrm{N}$ and $\mathbf{P}$ of the chalcopyrite Brillouin zone [6]. The lines between calculated energy levels were drawn taking into account the compatibility relations and following the energy band structures of $\mathrm{ZnGeAs}_{2}$ and $\mathrm{ZnGeP}_{2}$ obtained by imbedding the energy bands of their closest zincblende analogs ( $\mathrm{GaAs}$ and $\mathrm{GaP}$ ) into the chalcopyrite Brillouin zone.

The uppermost valence bands are similar in both types of band structure and are derived from the $\Gamma_{15}$ valence band maximum in $11 \mathrm{I}-\mathrm{V}$ zincblende crystals.

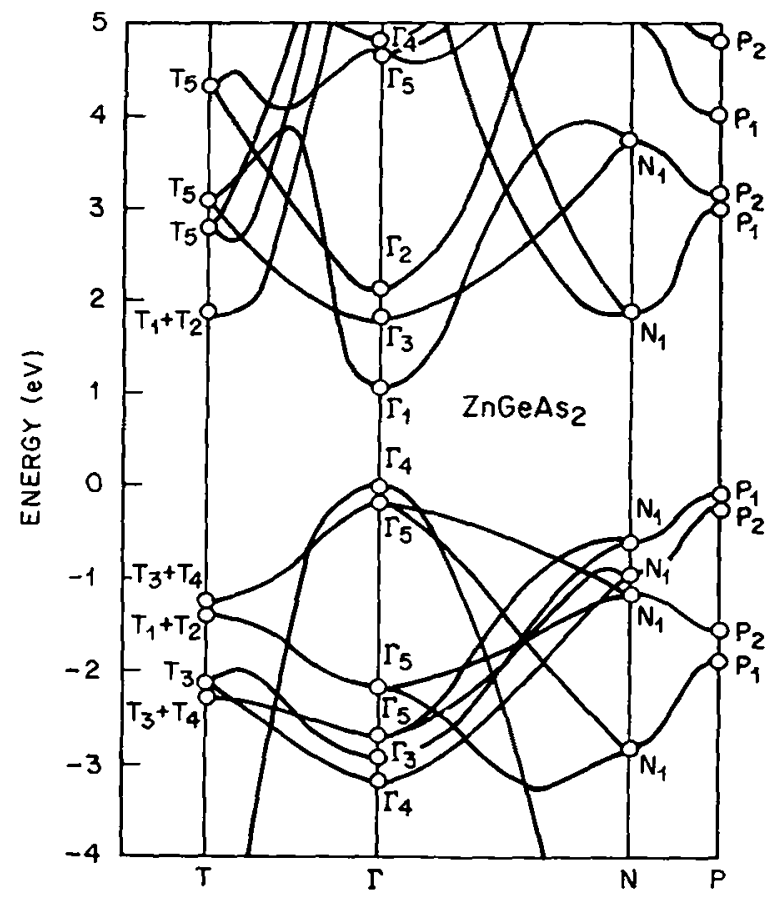

FIG. 1. - Energy band structure of $\mathrm{ZnGeAs}_{2}$ (after ref. [6]).

Due to the reduced symmetry of the chalcopyrite structure, the triple degeneracy of $\Gamma_{15}$ is lifted and the nondegenerate $\Gamma_{4}$ lies above the doubly degenerate $\Gamma_{5}$. This crystal field splitting $A_{\text {cf }}$ between $\Gamma_{4}$ and $\Gamma_{5}$ has been measured for several compounds and is plotted in figure 3 as a function of the uniaxial lattice compression $2-c / a$, where $c$ and $a$ are the lattice constants. It is apparent that the crystal field splitting of the uppermost valence bands is a linear function of $2-c / a$. Theoretical calculations [6] predict such a trend, but quantitative agreement is not obtained. Unfortunately a recent calculation [7] of the band structure of $\mathrm{ZnGeP}_{2}$ obtains the wrong sign of the crystal field splitting.

The lowest conduction band $\Gamma_{1}$ in $\mathrm{ZnGeAs}_{2}$ (Fig. 1) is derived from the $\Gamma_{1}$ conduction band in GaAs. The 


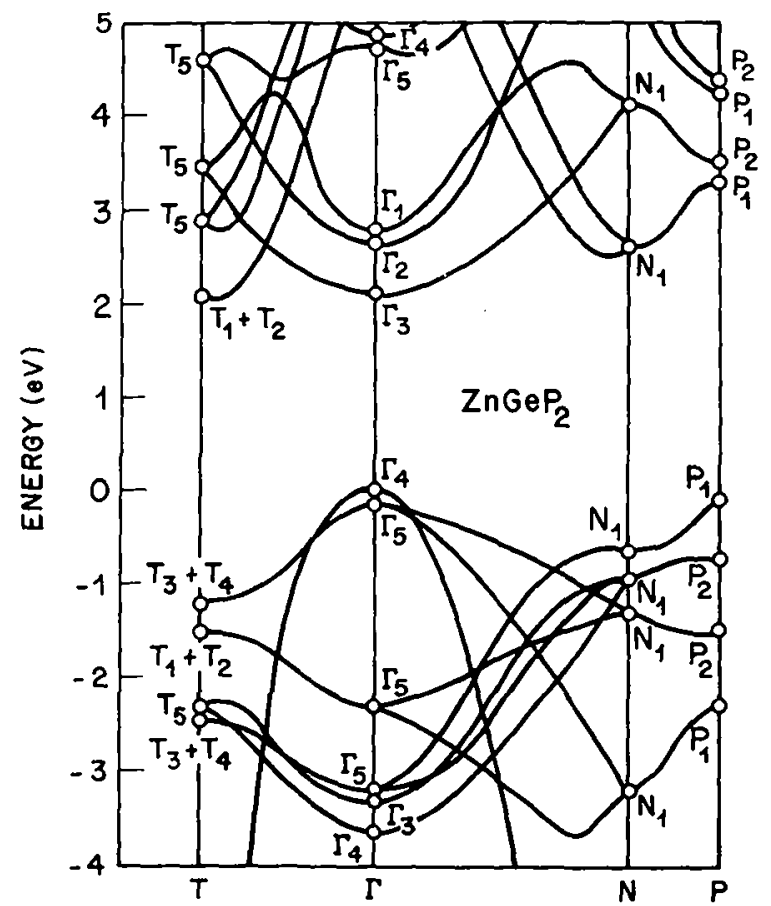

Fig. 2. - Energy band structure of $\mathrm{ZnGeP}_{2}$ (after ref. [6]).

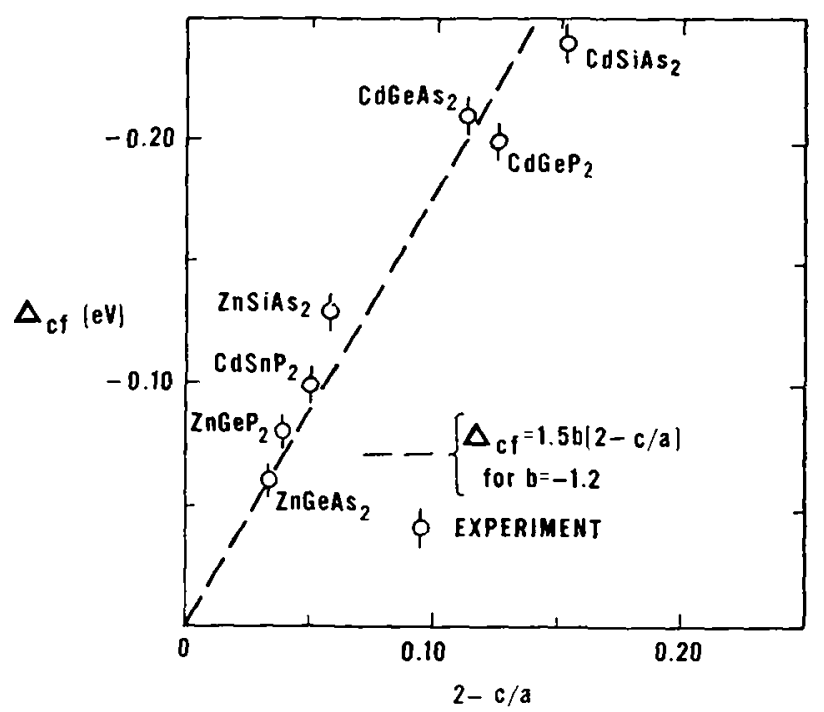

FIG. 3. - Crystal field splitting of the uppermost valence bands of several II-IV- $\mathrm{V}_{2}$ compounds as a function of the uniaxial lattice compression (after ref. [5]).

higher energy $\Gamma_{3}$ and $\Gamma_{2}$ conduction bands are derived from the $X_{1}$ and $X_{3}$ conduction bands in GaAs and are mapped into the $\Gamma$ point due to the smaller Brillouin zone of the chalcopyrite structure. The opposite ordering of the conduction bands is observed in $\mathrm{ZnGeP}_{2}$ (Fig. 2). Optical transitions between levels at the same point in the chalcopyrite Brillouin zone but derived from indirect transitions in the II-V analog have been termed pseudodirect [8] since their strengths will depend upon the differences in the cation pseudopotentials. Typically, pseudodirect transitions are about an order of magnitude weaker than direct transitions.

Direct optical transitions from $\Gamma_{4}$ and $\Gamma_{5}$ to the $\Gamma_{1}$ conduction band are allowed only for $\mathbf{E} / / \mathbf{z}$ and $\mathbf{E} \perp \mathbf{z}$ respectively, where $\mathbf{z}$ is the optic axis. Although these selection rules are relaxed somewhat when the double degeneracy of $\Gamma_{5}$ is lifted by spin-orbit interaction, the observed polarization properties of the three transitions labelled $\mathrm{A}, \mathrm{B}$ and $\mathrm{C}$ are quantitatively explained by a single, quasicubic model [5].

The electroreflectance spectrum [8] of the pseudodirect crystal $\mathrm{ZnSiP}_{2}$ is shown in figure 4 . The lowest

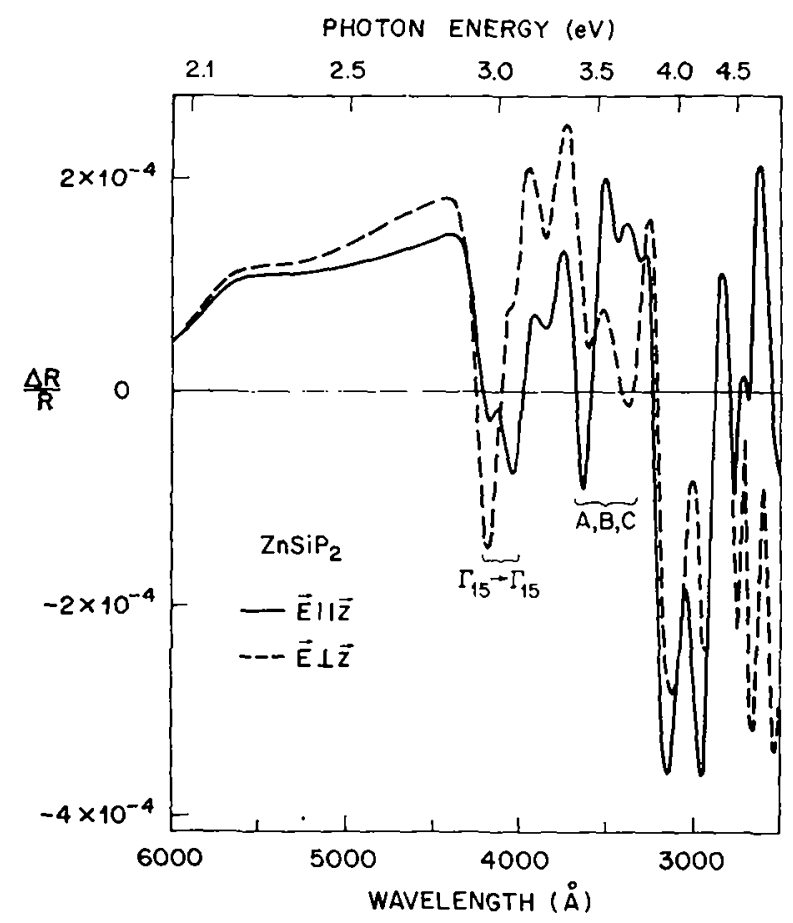

FIG. 4. - Electroreflectance spectrum of $\mathrm{ZnSiP}_{2}$ (after ref. [8]).

energy direct transitions near $3.0 \mathrm{eV}$ in figure 4 cannot be due to $\Gamma_{4}, \Gamma_{5} \rightarrow \Gamma_{1}$ transitions since the observed polarization dependences are opposite to those observed in other ternary crystals. We suggest instead that the structure observed near $3.5 \mathrm{eV}$ results from the A, B and $\mathrm{C}$ chalcopyrite gaps derived from $\Gamma_{15} \rightarrow \Gamma_{1}$ transition in zincblende crystals. The origin of the structure near $3.0 \mathrm{eV}$ is not certain, but it may be derived from $\Gamma_{15} \rightarrow \Gamma_{15}$ transitions in zincblende crystals. The strength of the $3 \mathrm{eV}$ structure renders as interpretation based upon pseudodirect transitions unlikely. Our suggested ordering for the conduction band levels (i. e. $\Gamma_{15}$ below $\Gamma_{1}$ ) conflicts with an energy band calculation [6] which predicts

$$
\left(\Gamma_{15} \rightarrow \Gamma_{1}\right) \approx 3.2 \mathrm{eV} \text { and }\left(\Gamma_{15} \rightarrow \Gamma_{15}\right) \approx 4.2 \mathrm{eV}
$$

A recent calculation [9] using a generalization of the dielectric theory of Phillips and Van Vechten [10] predicts a larger value for the fundamental band gap $\left(\Gamma_{15} \rightarrow \Gamma_{1}\right)=3.9 \mathrm{eV}$. A definitive determination of 
which structure in $\mathrm{ZnSiP}_{2}$ results from $\Gamma_{4}, \Gamma_{5} \rightarrow \Gamma_{1}$ transitions is possible by a study of these peaks in the electroreflectance spectra of $\mathrm{Zn}\left(\mathrm{Si}_{x} \mathrm{Ge}_{1-x}\right) \mathrm{P}_{2}$ or $\mathrm{ZnSi}\left(\mathrm{P}_{x} \mathrm{As}_{1-x}\right)_{2}$ alloys, since the band gaps in $\mathrm{ZnGeP}_{2}$ and $\mathrm{ZnSiAs}_{2}$ are well established [5]. The interpretation of the electroreflectance spectrum of $\mathrm{ZnSiP}_{2}$ could also be established by a full-zone calculation of the optical properties as has been done for binary compounds by many workers.

3. I-III-VI I $_{2}$ compounds. - In an earlier review [3] it was shown that the uppermost valence bands of a I-III-VI ${ }_{2}$ compound are profoundly influenced by the proximity of noble metal d-levels in the valence band. The consequence of this interaction are easily described with the simple sketch shown in figure 5. The fivefold
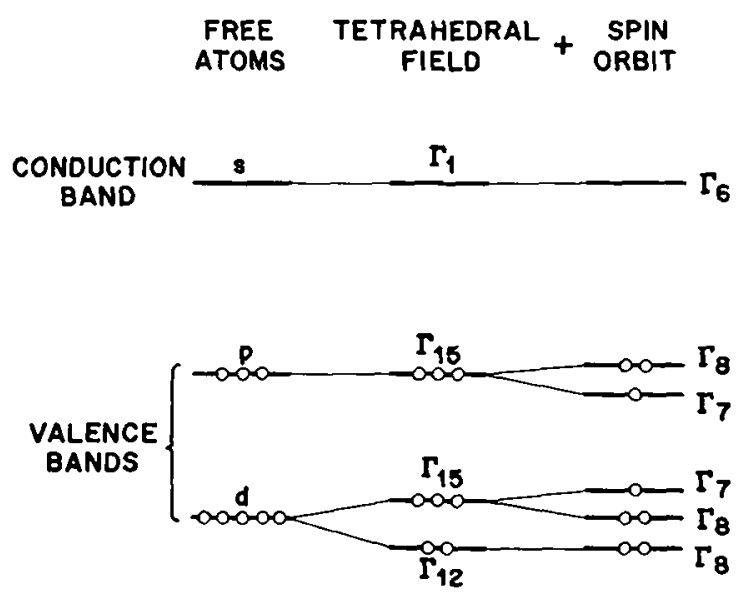

FIG. 5. - Qualitative sketch of the expected behavior of d-levels in a tetrahedral field (after ref. [3]).

degenerate d-levels split into a threefold $\Gamma_{15}$ and a twofold $\Gamma_{12}$ in a tetrahedral field ; p-levels transform as $\Gamma_{15}$. Allowing for spin splits the p-like $\Gamma_{18}$ into a doublet $\Gamma_{8}$ above a singlet $\Gamma_{7}$, whereas the d-like $\Gamma_{15}$ splits into a doublet $\Gamma_{8}$ below a singlet $\Gamma_{7}$. These results follow simply from a diagonalization of the $1 . \mathrm{s}$ matrices [11]. Interaction between the $\Gamma_{15}$ levels in figure 5 will have two effects. The uppermost $\Gamma_{1 s}$ will be raised to higher energy, i. e., the band gap will be reduced, and the spin-orbit splitting of the uppermost valence bands will be reduced, because the negative spin-orbit parameter $\left(\Gamma_{8}-\Gamma_{7}\right.$ splitting) of the d-levels partially cancels the positive spin-orbit parameter of p-levels. A quantitative estimate of the p-d hybridization can therefore be determined from the measured spin-orbit splitting of the uppermost valence bands.

In figure 6 is plotted for each I-III-VI 2 compound, the reduction of the energy gap relative to its binary analog, and the fractional d-like character of the uppermost valence bands. The noticeable correlation between these quantities is taken as proof for the validity of p-d hybridization in I-III-VI ${ }_{2}$ compounds.

Recent X-ray photoemission studies $[12,13]$ (XPS)

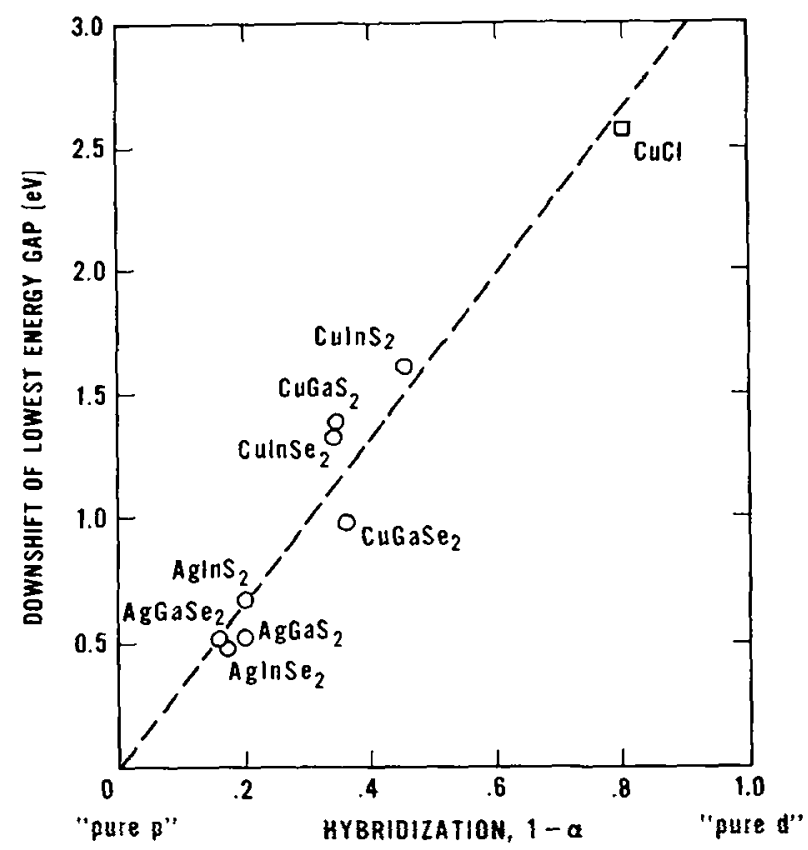

FiG. 6. - Plot of the reduction of the energy gap in a ternary compound relative to its binary analog, as a function of the d-like character of the uppermost valence bands determined from the observed spin-orbit splittings (after ref. [3]).

have substantiated the essential validity of this concept. Figure 7 shows the XPS valence band spectra of $\mathrm{CuInS}_{2}, \mathrm{CuGaS}_{2}, \mathrm{CuAlS}_{2}$ and $\mathrm{AgGaS}_{2}$ obtained with $\mathrm{MgK} \alpha_{1,2}$ (1 $253.6 \mathrm{eV}$ ) excitation. The spectra in figure 7 show a striking contrast between the $\mathrm{Cu}$ compounds and the Ag-compounds in that each $\mathrm{Cu}$-compound has the major peak in the low-bindingenergy region of the spectrum and the Ag-compound in the high-binding-energy region. Since d-states generally offer intense peaks in an XPS spectrum, the major peaks in figure 7 may be attributed to the states which consist mainly of noble metal d-wavefunctions. These quantitative results agree with the data in figure 6 which show the uppermost valence bands in $\mathrm{Cu}$-compounds to be considerably more d-like than those in $\mathrm{Ag}$ compounds. Moreover the energies of the d-peaks for the $\mathrm{Cu}$-compounds in figure 7 agree with values deduced from optical transitions attributed to transitions originating on the $\mathrm{d}$-levels themselves $[14,15]$. The larger binding energy of d-levels in $\mathrm{AgGaS}_{2}$ (Fig. 7) agrees with the absence of any observed optical transitions from d-levels in this compound.

Since the optical and $X$-ray experiments substantiate the importance of noble-metal d-levels in I-III-VI compounds, a theoretical calculation including d-levels would be of considerable interest. The only existing calculation for I-III-VI $\mathrm{V}_{2}$ compounds [16] ignores d-levels and as a result fails to explain most of the experimental observations in these compounds [14]. A theoretical calculation [17] of nonlinear optical suscep- 


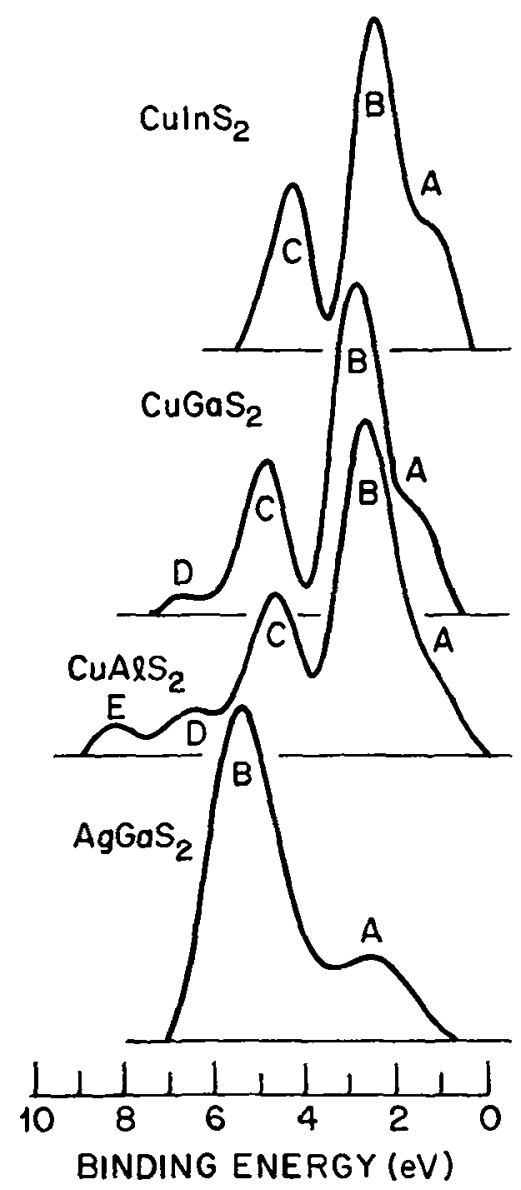

FIG. 7. - X-ray photoelectron spectra of the valence bands in $\mathrm{CuInS}_{2}, \mathrm{CuGaS}_{2}, \mathrm{CuAlS} 2$ and $\mathrm{AgGaS}_{2}$ (after ref. [13]).

tibilities of these compounds found d-band contributions to be significant.

There is another interesting observation in a I-III$\mathrm{VI}_{2}$ compound which may be amenable to theoretical calculation, namely the vanishing crystal field splitting of the uppermost valence bands in $\operatorname{CuInS}_{2}[18,19]$. The wavelength-modulated reflectivity (WMR) spectrum of

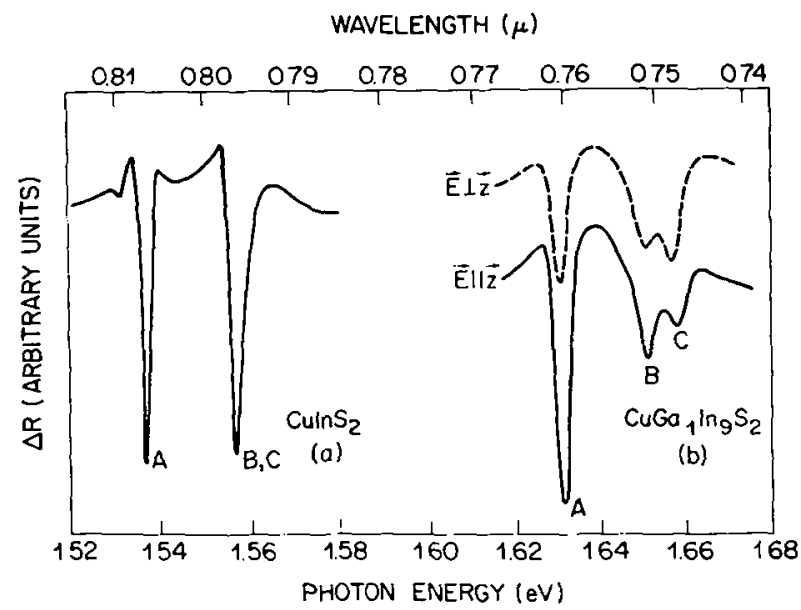

FIG. 8. - Wavelength-modulated reflectance spectra at $2 \mathrm{~K}$ of (a) $\mathrm{CuInS}_{2}$ and (b) $\mathrm{CuGa}_{0.1} \mathrm{In}_{0.9} \mathrm{~S}_{2}$ (after ref. [18] and [19]).
$\mathrm{CuInS}_{2}$ is shown in figure $8 a$ and consists of two structures separated by $19 \mathrm{meV}$. Since this spectrum is independent of the polarization of the incident light, the $19 \mathrm{meV}$ splitting results from spin-orbit interaction. An earlier exciton-oscillator fit to the reflectivity of $\mathrm{CuInS}_{2}$ required that the oscillator strength of the higher energy transition be twice that of the lower energy transition [20]. Consequently, it was concluded that the doubly degenerate valence band $\mathrm{B}, \mathrm{C}$ lay below the nondegenerate band $\mathrm{A}$. As shown in figure $8 b$, the addition of slight amounts of Ga to $\mathrm{CuInS}_{2}$ shifts the spectrum to higher energies, lifts the degeneracy of the $\mathrm{B}, \mathrm{C}$ transitions, and produces an observable polarization dependence in the WMR spectrum. From the splittings of the three structures in figure $8 b$, we derive spin-orbit and crystal field parameters of $-22 \mathrm{meV}$ and $-11 \mathrm{meV}$ respectively.

The crystal-field parameters of several alloys are plotted as a function of composition in figure $9 a$ [21]. It is apparent that the crystal-field splitting is nearly linear in $x$ varying from zero in $\mathrm{CuInS}_{2}$ to $-130 \mathrm{meV}$ in $\mathrm{CuGaS}_{2}$. The vanishing of $\Delta_{\mathrm{cf}}$ in $\mathrm{CuInS}_{2}$ is of interest since the built-in lattice distortion (Fig. $9 b$ ) should lead to an observable $\Delta_{\mathrm{cf}}$. In earlier studies of chalcopyrite crystals (see Fig. 3) for which $2-c / a$ was large and positive, $\Delta_{\mathrm{cf}}$ was negative and approximately proportional to $2-c / a$. Since $\mathrm{CuInS}_{2}$ has a negative $2-c / a$, yet $\Delta_{\mathrm{cf}} \approx 0$, the expected $\Delta_{\mathrm{cf}}$ due to this elongation must be cancelled by a negative $\Delta_{\mathrm{cf}} \approx-35 \mathrm{meV}$ due to the internal distortion (the deviation from perfect-tetrahedral bonding) and the restructuring of the Brillouin zone as a consequence of
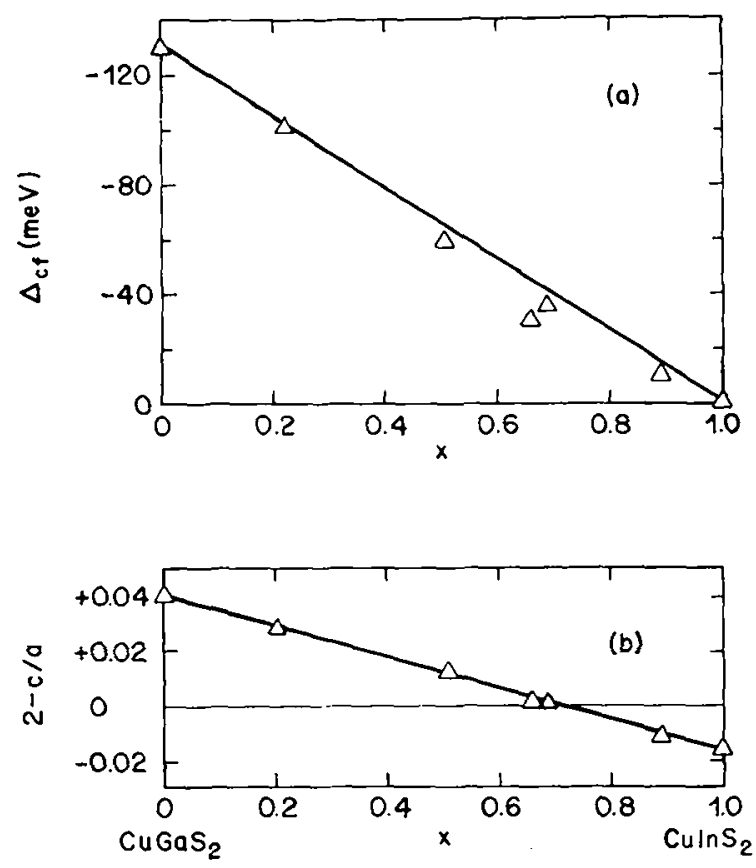

FIG. 9. - Compositional dependence of (a) the crystal-field parameter $\Delta_{c f}$, and $(b)$ the built-in lattice distortion $2-c / a$, in the system $\mathrm{CuGa}_{1-. x} \operatorname{In}_{x} \mathrm{~S}_{2}$ (after ref. [18] and [19]). 
the two different cations. This phenomenon has yet to be observed in any other ternary crystal.

4. Conclusion. - In conclusion we emphasize the need for sophisticated energy band calculations and full-zone calculations of the optical properties of chalcopyrite compounds. In Section 2 we discussed the pseudodirect compound $\mathrm{ZnSiP}_{2}$ whose anomalous band structure could be definitively interpreted by such calculations. Higher energy transitions in figure 4 and many other crystals $[1,4,5]$ await such calculations for definitive interpretation.
The importance of noble metal d-bands in I-III-VI compounds is clear from electroreflectance and X-ray photoemission experiments. Band structure calculations incorporating these d-levels are needed. The assignment of certain electroreflectance structures to transitions from the d-levels themselves could readily be verified by full-zone calculations of the optical properties of I-III-IV 2 compounds.

Acknowledgments. - We are grateful to E. Buehler, H. M. Kasper, B. Tell and J. H. Wernick for their collaboration in most of the work reviewed here.

\section{References}

[1] SHAY, J. L., Proceedings of the 11 th International Conference on the Physics of Semiconductors, Warsaw, Poland (PWN-Polish Scientific Publishers) 1972, p. 787.

[2] Benidorius, R. A., Krivaite, G. Z., Shileika, A. Yu., Karavaev, G. F. and Chaldyshev, V. A., Proceedings of the 11th International Conference of the Physics of Semiconductors, Warsaw, Poland (PWN-Polish Scientific Publishers) 1972, p. 838.

[3] Shay, J. L. and Tell, B., Surf. Sci. 37 (1973) 748.

[4] ShILejKa, A., Surf. Sci. 37 (1973) 730.

[5] Shay, J. L. and Wernick, J. H., Ternary Chalcopyrite Semiconductors : Growth, Electronic Properties, and Applications (Pergamon) 1975.

[6] Poplavnor, A. S., Polygalov, Yu. I. and Chaldyshev, V. A., Izv. VUZ. Fiz. 12 (1970) $58 ; 13$ (1970) 95;13(1970) 17 [Soviet Physics Journal (1972) 1415; 13 (1973) 766; 13 (1973) 849].

[7] De Alvarez, C. V. and Cohen, M. L., Phys. Rev. Lett. 30 (1973) 979 ;

De Alvarez, C. V., Cohen, M. L., Kohn, S. E., Petroff, Y. and Shen, Y. R., Phys. Rev. B 10 (1974) 5175 .

[8] Shay, J. L., Buehler, E. and Wernick, J. H., Phys. Rev. B 3 (1971) 2004 ;

Shay, J. L., Tell, B., Buehler, E. and Wernick, J. H., Phys. Rev. Lett. 30 (1973) 983.

[9] Pasfmann, L., Phys. Stat. Sol. (b) 56 (1973) K 69.
[10] Van Vechten, J. A. and Phillips, J. C., Phys. Rev. 82 (1970) 2160.

[11] Shindo, K., Morita, A. and Kaminura, H., J. Phys. Soc. Japan 20 (1965) 2054.

[12] Luciano, M. J. and Vesely, C. J., Appl. Phys. Lett. 23 (1973) $60 ; 23$ (1973) 453.

[13] Syozo Kono and Makoto Okusawa, J. Phys. Soc. Japan 37 (1974) 1301.

[14] Shay, J. L. and Kasper, H. M., Phys. Rev. Lett. 29 (1972) 1162.

[15] Gan, J. and Tauc, J., Solid State Commun. 15 (1974) 605.

[16] Poplaynor, A. S. and Polygalov, Yu. I., Izv. Akad. Nauk SSSR, Neorg. Mater. 7 (1971) 1706, 1711 [Inorg. Mater. (USSR) 7 (1971) 1527, 1531].

[17] Levine, B. F., Phys. Rev. B 7 (1973) 2591.

[18] Shay, J. L., Tell, B. and Kasper, H. M., Proceedings of the 12 th Int. Conf. on the Physics of Semiconductors, Sluttgart, 1974, B. G. Teubner, p. 311.

[19] Tell, B., Shay, J. L., Kasper, H. M. and Barns, R. L., Phys. Rev. B 10 (1974) 1748.

[20] Shay, J. L., Teld, B., Kasper, H. M. and Schiavone, L. M., Phys. Rev. B 5 (1972) 5003.

[21] Compositions were determined from the measured lattice constants using Vegards law which has been shown to be valid in this system by Yamamoto, $\mathrm{N}$. and MiyauCHI, T., Japan J. Appl. Phys. 11 (1972) 383. 